\title{
Research on the Real-time Inventory Data Acquisition Management System and Technology of Railway Fuel
}

\author{
Yanxia Wang ${ }^{1, \text { a }}$, Wenxiu Zhao ${ }^{1, \mathrm{~b}}$, Haitao Zhang ${ }^{2, \mathrm{c}}$ and Longhai $\mathrm{Xu}^{2, \mathrm{c}}$ \\ ${ }^{1}$ MOE Key Laboratory for Urban Transportation Complex Systems Theory and Technology, \\ Beijing Jiaotong University, Beijing 100044, China; \\ ${ }^{2}$ CRM E-Commerce Tech.Co.,Ltd, Beijing 100088, China. \\ ayanxiaw0326@126.com, ${ }^{b} 15120929 @$ bjtu.edu.cn, cczhanght@crmec.com.cn
}

Keywords: Inventory management; real-time data acquisition; data acquisition process; data exchange process; railway fuel.

\begin{abstract}
As the power source of locomotive, railway fuel is the main power of railway transportation. The real-time inventory data collection of railway fuel is of great significance for the main body of the railway fuel supply chain to control the inventory dynamics and manage the fuel stock. On the basis of inventory management process of railway, the paper analyzes the function of the real-time inventory data acquisition management system, designs the data acquisition process and data exchange process and the intelligentized acquisition method of railway fuel inventory data.
\end{abstract}

\section{Introduction}

With the rapid development of railway passenger and freight, railway fuel inventory management becomes more and more important. The real-time acquisition of railway fuel inventory data is useful to master the dynamic inventory, reduce operating costs and ensure the railway transportation. Deng Zaixiong et al. study on the hardware direct access, double buffer drawing display, direct access to the database and other solutions to obtain real-time data [1]. The data acquisition and monitoring system of temperature and inventory of oil tank in gas station is established by using the special data acquisition module, which can realize the real-time monitoring of oil products in all aspects [2]. Based on ARM10 embedded motherboard, Wang Xiancheng applies sensors, signal conditioning modules and PC104 acquisition board to design data acquisition system for diesel engine technical state, which can meet the requirements of real-time acquisition with low precision [3]. LI Dong et al. study EasiDEF: A Horizontal Lightweight Data Exchange Protocol for Internet of Things [4]. Supported by the national support program, China Railway Materials Commercial Corp and Beijing Jiaotong University study on the real time data acquisition and management system of railway fuel inventory data. This paper introduces the data acquisition process, data exchange process and intelligentized acquisition method of railway fuel inventory data.

\section{Process Analysis of Railway Fuel Inventory Management}

Railway fuel inventory management is not only an important daily work for the locomotive depot, but also one of the important basis for the financial settlement. Besides, it is a source to master basic inventory data for different subjects in the supply chain. Inventory management mainly includes daily check, density statistics, statistical analysis of profit and loss, safety management and so on. Fig.1 shows the inventory management process of railway fuel. 


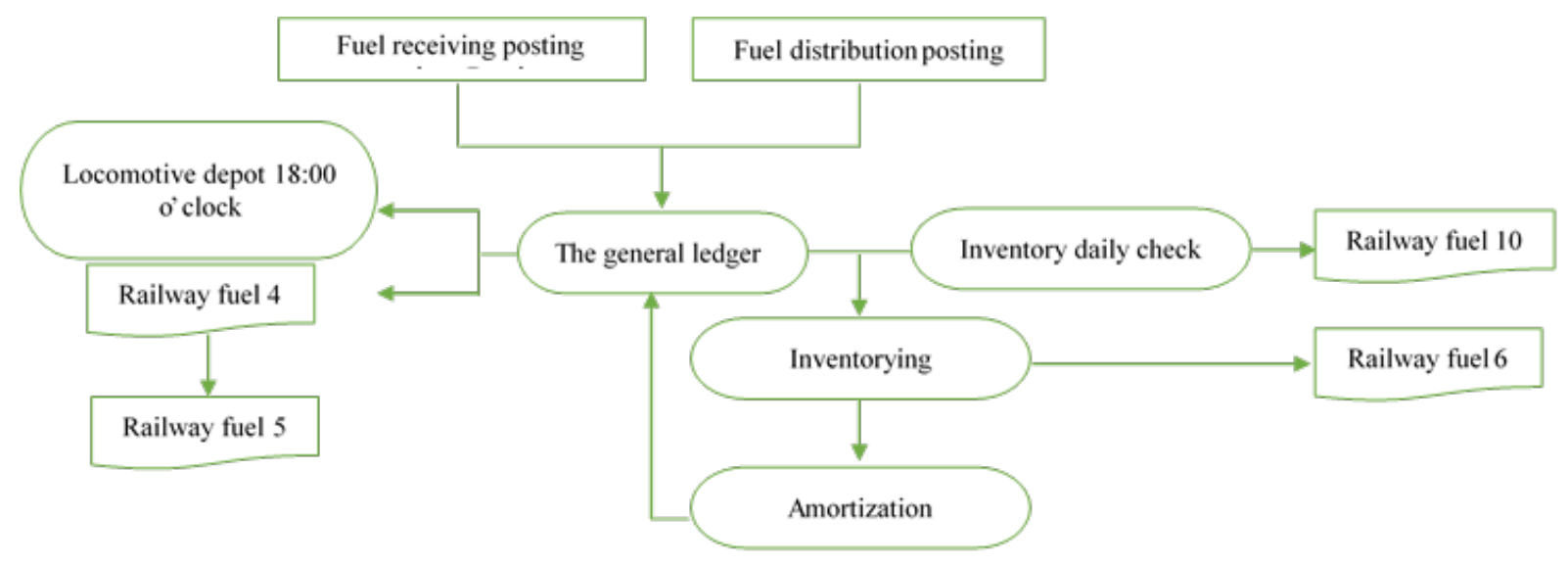

Fig. 1 The process of inventory management

\section{Function Analysis of the Real-time Inventory Data Acquisition Management System}

The real-time data acquisition and exchange system consists of level gauge, industrial equipment, application software and power, signal routing and other auxiliary equipments, which can be used in daily oil tank monitoring. The features of the system are as follows.

(1) automation and intelligentization of the measurement testing.

(2) high-precision and high-reliability testing.work.

(3) easy to operate and low-intensity.

The functions of real-time data acquisition and exchange system are as follows:

(1) real-time acquisition of storage state of tank and reservoir;

(2) automatic calculation of the fuel amount of tank and reservoir;

(3) real-time detection and grading alarm for index like fluid levels, water, temperature, inventory etc.

(4) save historical monitoring data, upload the monitoring data to the central application and receive instructions issued by the central application;

(5) the system status alarm, fault tolerance and fault oriented security mechanisms.

The functions are shown in Table 1 below.

Table 1 The functions of the real-time inventory data acquisition management system

\begin{tabular}{c|c}
\hline Function & Subfunction \\
\hline \multirow{4}{*}{ Data acquisition } & Inventory data acquisition \\
\cline { 2 - 2 } & Parameter setting \\
\cline { 2 - 2 } & Parameteracquisition and implementation \\
\cline { 2 - 2 } & Data acquisition of fuel gun \\
\cline { 2 - 2 } & Parameter setting of fuel gun \\
\hline \multirow{4}{*}{ Data exchange } & Data acquisition of IC card \\
\cline { 2 - 2 } & Parameter setting of IC card \\
\cline { 2 - 2 } & Information producer management \\
\cline { 2 - 2 } & Information producer allocation \\
\cline { 2 - 2 } & Information operator management \\
\cline { 2 - 2 } System & Information operator allocation \\
\cline { 2 - 2 } management & Information transfer management \\
\cline { 2 - 2 } & Information transfer allocation \\
\hline
\end{tabular}




\section{Design of the Data Acquisition Process and Data Exchange Process}

\subsection{Design of the Data Acquisition Process.}

The data acquisition process is designed as follows:

(1) The server in locomotive depot runs program of inventory acquisition;

(2) The acquisition program reads data including temperature and fluid levels from COM regularly ( such as 1 minute );

(3) Based on the data collected, compare the data change. If the change exceeds threshold level, the data should be sent to the acquisition server;

(4) If the change doesn't exceed the threshold, the current data should be sent to the acquisition serve periodically ( such as 30 minutes);

(5) The acquisition server receives the data and saves it into the database;

(6) If the data needs to be switched into the other end, then calls the data exchange process.

\subsection{Design of the Data Exchange Process.}

The data exchange process is designed as follows:

(1) Set up the Information Producer A, and turn the data into Information Package;

(2) Set up Information Transfer Service C;

(3) Set up Information Operator D;

(4) Set up the relation between Information Transfer Service C and Information Operator D;

(5) Set up Information Transfer Service B, and set C as receiver;

(6) A send the Information Package to B, B send it to C, and C send it to D;

(7) After receiving the Information Package, D unblocks the data.

The data should be transferred and shared in a way of pipeline between system and database[5], which can be seen in Fig.2.

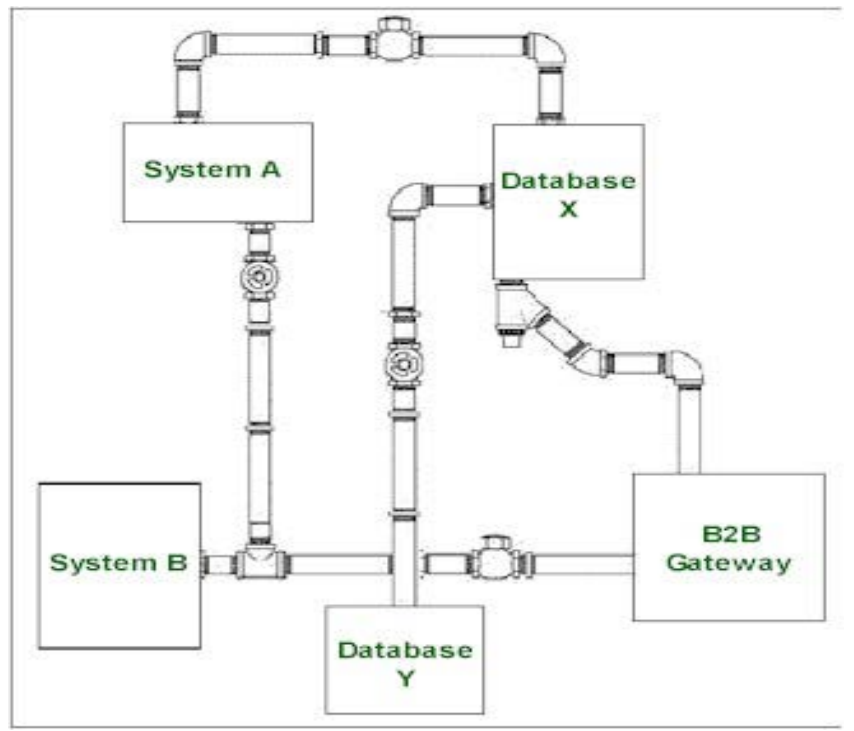

Fig. 2 The pipelines for data exchange

\section{The Intelligentized Acquisition Method of Railway Fuel Inventory Data}

The real-time intelligentized acquisition of storage tank temperature, liquid level, water level and other related inventory information includes the content of two following respects concretely [6]:

(1) data acquisition for reservoir-level equipment

Fig.3 shows the data acquisition for reservoir-level equipment during the interval period. 


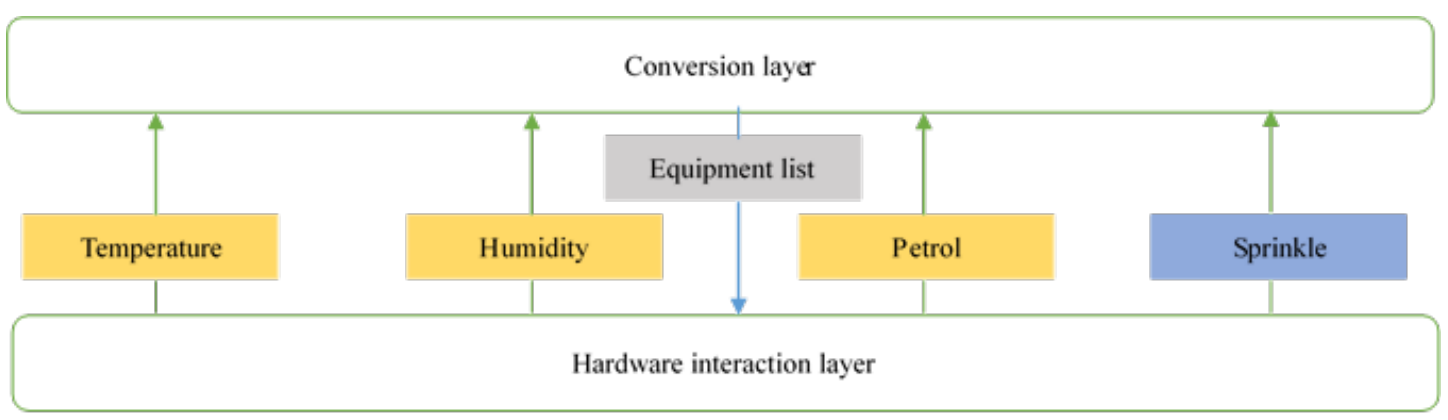

Fig. 3 Data acquisition for reservoir-level equipment

The structure in Fig. 3 is the parameter interface of reservoir-level equipment, which provides the kernel layer with staScan method to call parameters. Besides, based on the information of each device in this structure, the equipment layer will undertake communications and process the signals correspondingly.

(2) data acquisition for storage tank level equipment

Fig.4 shows the data acquisition like temperature, liquid level and water level for storage tank level equipment during the interval period.

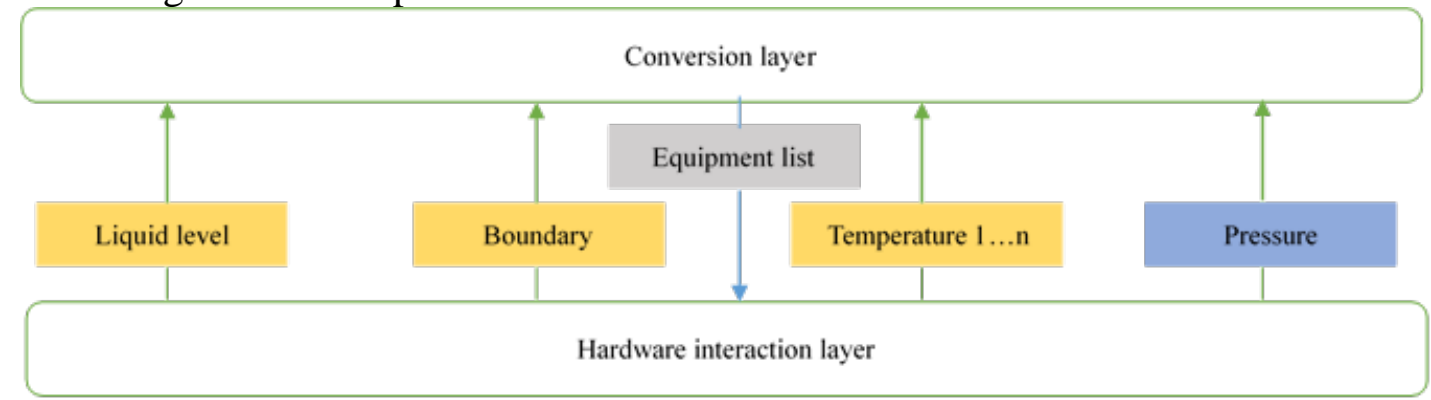

Fig. 4 Data acquisition for storage tank level equipment

The structure in Fig. 4 is the feedback interface of checking data, where defines the signals needed to be collected and the arrays exceptions in the course of the interaction. Besides, the feedback data should be in accord with the equipment list, for example, if the liquid level equipment hasn't been defined, the liquid level can be null.

\section{Conclusion}

The paper analyzes the management process of railway fuel inventory and determines the functions of the real-time inventory data acquisition management system, then further designs the data acquisition process and data exchange process. In addition, the real-time intelligent acquisition has been refined into reservoir-level equipment and storage tank level equipment, which provides support for railway fuel inventory management.

\section{Acknowledgment}

The paper is sponsored by National Science-technology Support Plan Projects "Research and Application of the Third Party Logistics Service Platform of Railway Oil Supply Chain” (No. 2014BAH23F02) and "Research of Real-time Monitoring, Tracing and Management Technology of Supply Chain Logistics” (No. 2014BAH23F01).

\section{References}

[1]. Deng Zaixiong and Zhao Huiqing, "On some problems in the software development of realtime data acquisition and processing system” $[\mathrm{J}]$, Journal of Beijing University of Chemical Technology (Natural Science Edition). Beijing, vol. 31(6), pp. 101-103, 2004. 
[2]. Wang Xiao, “On-Line Monitoring System for Oil Temperature and Profit and Loss for Gasline Tank at Gasline Station,” Automation in Petro-Chemical Industry. vol. 50(2), pp.35-38, 2014.

[3]. Wang Xiancheng, Zhang Genliang, Hemu and Jiang Li, "Design of State Data Real-time Acquisition System for Vehicle Diesel Engine,” Vehicle Engine. vol. (4), pp.23-26, 2011

[4]. HOU Chen-Da, LI Dong, QIU Jie-Fan, CUI Li, “EasiDEF: A Horizontal Lightweight Data Exchange Protocol for Internet of Things”[J], Chinese Journal of Computers, 2015, 38(3)

[5]. BU Qian, YU Peng-fei, ZHANG Wei-cun, ZHANG Lin, "Model for product data exchange based on ontology”[J], Computer Integrated Manufacturing Systems, 2009, 15(5)

[6]. REN Kaiyin, KONG Zhen, YE Min, "Design and Implementation of General Purpose Data Exchange System”[J], Automation of electric Power Systems, 2009, 33(20) 